\title{
Involvement of PAL, C4H, and 4CL in Chilling Injury-induced Flesh Lignification of Loquat Fruit
}

\author{
Xue Li, Chen Zang, Hang Ge, and Jing Zhang \\ Zhejiang Provincial Key Laboratory of Horticultural Plant Integrative \\ Biology, Zhejiang University, Zijingang Campus, Hangzhou 310058, \\ People's Republic of China \\ Donald Grierson \\ Plant and Crop Sciences Division, School of Biosciences, University of \\ Nottingham, Sutton Bonington Campus, Loughborough, LE12 5RD, UK
}

Xue-ren Yin ${ }^{1}$ and Kun-song Chen

Zhejiang Provincial Key Laboratory of Horticultural Plant Integrative Biology/The State Agriculture Ministry Laboratory of Horticultural Plant Growth, Development and Quality Improvement, Zhejiang University, Zijingang Campus, Hangzhou 310058, People's Republic of China

Additional index words. loquat (Eriobotrya japonica), postharvest lignification, 4-coumate: coenzyme A ligase, cinnamate 4-hydroxylase, L-phenylalanine ammonia lyase, chilling injury

\begin{abstract}
Loquat (Eriobotrya japonica) is a model fruit for investigating flesh lignification during storage and response to chilling injury. However, the investigations of enzymes and coding genes and loquat fruit lignification under low-temperature storage are still limited. Here, the activity and transcript levels of up-stream enzymes of the phenylpropanoid pathway, including L-phenylalanine ammonia-lyase (PAL), cinnamate 4-hydroxylase $(\mathrm{C} 4 \mathrm{H})$, and 4-coumarate:coenzyme A ligase $(4 \mathrm{CL})$, were investigated. The results indicated that activity of these enzymes was positively correlated with loquat fruit lignification and suppression of these increases by heat treatment (HT) and lowtemperature conditioning (LTC) significantly alleviated loquat fruit lignification. Coding genes for these enzymes were subsequently isolated based on information from an RNAseq database and expression of $E j 4 C L 1$ was found to be the most responsive to low temperature and inhibition by HT and LTC treatment, whereas the other genes were less responsive to these treatments. Furthermore, function of Ej4CL1 was analyzed by transient overexpression in tobacco leaves, where it stimulated lignin accumulation. Ej4CL1 may be a key candidate that involved in CI-related loquat fruit lignification.
\end{abstract}

Texture is an important quality trait for fruit (Li et al., 2010). Postharvest softening usually occurs in most fruit, but lignification is an uncommon phenomenon occurring in ripening fruit, which is usually accompanied by increased fruit firmness, leathery pulp, internal browning, and decreased juiciness (Lin et al., 1999). Loquat (Eriobotrya japonica Lindl.) fruit show substantial flesh lignification during postharvest storage, and this is more severe when fruit are subjected to cold storage (Cai et al., 2006a, 2006b). Loquat fruit lignification can be significantly alleviated by various treatments, such as LTC (Cai et al., 2006b), HT (Xu et al., 2014; Zeng et al., 2015), 1-methylcyclopropene (1-MCP) (Cai et al., 2006c), Methyl jasmonate

\footnotetext{
Received for publication 26 Aug. 2016. Accepted for publication 9 Nov. 2016.

This research was supported by the National Basic Research Program of China (2013CB127104), National Natural Science Foundation of China (no. 31372041) and the Natural Science Foundation of Zhejiang Province, China (LR16C150001). ${ }^{1}$ Corresponding author. E-mail: xuerenyin@zju. edu.cn.
}

(MeJA) (Cai et al., 2011; Cao et al., 2012), and Nitric oxide (NO) (Xu et al., 2012). Thus, loquat fruit is an ideal material for investigating fleshy fruit lignification.

Generally, lignification is caused by the accumulation of lignin in plant cell walls and the biosynthesis of lignin has been widely studied in the model plant Arabidopsis, woody trees and some other crops (Vanholme et al., 2010; Zhao and Dixon, 2011). Monolignol formation is catalyzed by a series of enzymes in the phenylpropanoid pathway, such as PAL, C4H, 4CL, hydroxycinnamoylCoA:NADPH moxidoreductase, cinnamyl alcohol dehydrogenase (CAD), etc (Barros et al., 2015; Bonawitz and Chapple, 2012). Many of the coding genes for these enzymes have been functionally verified to be involved in lignin biosynthesis, for instance, 4CL antisense lines in Arabidopsis showed lower lignin content, and altered lignin composition compared with wide-type plants (Lee et al., 1997). Similarly, both PAL and $\mathrm{C} 4 \mathrm{H}$ suppression lines of tobacco produced lower lignin content and altered lignin composition (Sewalt et al., 1997).
In loquat fruit, the relationship between biosynthetic enzymes and lignification showed CAD activity was closely related to lignin contents of different cultivars (Shan et al., 2008). Moreover, CAD activities, as well as EjCAD1 expression, was also positively correlated with low temperature-induced loquat fruit lignification (Shan et al., 2008). Recently, EjCCOAOMT was newly isolated from loquat fruit and expression also shown to be induced by low temperature (Liu et al., 2015). As mentioned above, various enzymes and their coding genes within phenylpropanoid pathway contributed to lignin biosynthesis. However, the roles of other components within phenylpropanoid pathway for loquat fruit lignification remain unclear.

Recently, several transcription factors have been reported to be involved in lignin regulation in loquat fruit, including EjMYB1, EjMYB2 (Xu et al., 2014), EjAP2-1 (Zeng et al., 2015), EjNACl (Xu et al., 2015), and EjHSF3 (Zeng et al., 2016). All of these transcription factors significantly influenced promoters of genes-encoded PAL and 4CL of the phenylpropanoid pathway, supporting the potential roles of multiple enzymes in loquat lignification regulation. Thus, in the present research, activities of PAL, C4H, and $4 \mathrm{CL}$ were analyzed in response to low temperature, LTC and HT in loquat cv. Luoyangqing (LYQ). Based on our previous results (Shan et al., 2008) and using an RNA-seq database, some new coding genes for these enzymes were isolated, their expression studied, and transient overexpression analysis was performed on $N$. tabacum to verify the role of Ej4CL1 in lignin biosynthesis.

\section{Materials and Methods}

Fruit materials and treatments. The fruit materials used in the present research were derived from our previous publication $(\mathrm{Xu}$ et al., 2014). In brief, 'LYQ' loquat fruit were collected at commercial maturity from an orchard at Luqiao, Zhejiang, China in 2011. Fruit without disease or mechanical wounding were selected and divided into three groups for treatments. Three treatments are as follow: 1) Control, stored at $0{ }^{\circ} \mathrm{C} ; 2$ ) LTC, storage at $5{ }^{\circ} \mathrm{C}$ for $6 \mathrm{~d}$ and then transferred to $0{ }^{\circ} \mathrm{C} ; 3$ ) heat treatment (HT), treated at $40{ }^{\circ} \mathrm{C}$ for $4 \mathrm{~h}$ and then transferred to $0{ }^{\circ} \mathrm{C}$. At each sampling point, three replicates, each of five fruits, were sampled without skins and kernels. The flesh tissues were cut and frozen in liquid nitrogen immediately and stored at $-80{ }^{\circ} \mathrm{C}$.

Enzyme activity assays. Activities of three enzymes within the phenylpropanoid pathway were measured according to the previously published methods (Knobloch and Hahlbrock, 1975; Koukol and Conn, 1961; Lamb and Rubery, 1975). One unit of enzyme activity was defined as the amount of enzyme that caused a change of 0.01 in absorbance per min. Protein levels were measured using the method of Bradford 
(1976). Three biological replicates were performed for enzyme activity measurement.

Gene isolation and analysis. Two EjPAL genes and five $E j 4 C L$ genes were previously isolated (Shan et al., 2008; Xu et al., 2014). Using RNA-seq database, two $\mathrm{EjC} 4 \mathrm{H}$ genes and six $E j 4 C L$ genes were newly isolated from loquat. Full-length sequences were obtained from partial sequences using SMART RACE cDNA Amplification kit (Clontech), with primers listed in Table 1. Alignment of the deduced amino acid sequences was performed in ClustalX (v 1.81) and the phylogenetic tree was constructed using Figtree (v1.4.2).

RNA extraction and $c D N A$ synthesis. Total RNA was extracted following the previously published methods (Shan et al., 2008). The contaminating DNA was digested using TURBO DNA-free kit (Ambion). The first strand of cDNA was synthesized with iScript cDNA Synthesis Kit (Bio-Rad) following the manufacturer's protocol. Three batches of RNA were isolated as three biological replicates for each time point. Diluted cDNA was used as template of realtime polymerase chain reaction (PCR).

Real-time PCR. Oligonucleotide primers for real-time PCR were designed from the untranslated region of individual genes and are listed in Table 2. Loquat actin was used as an internal standard (Shan et al., 2008). Realtime PCR was performed in a total volume of $20 \mu 1$ with $10 \mu 12 \times$ LightCycler 480 SYBR Green I Master Mix (Roche), $6 \mu$ l PCR-grade water, $2 \mu \mathrm{cDNA}$, and $2 \mu \mathrm{l}$ primer mixture on a LightCycler 480 instrument (Roche). The PCR program included initiation at $95^{\circ} \mathrm{C}$ for 5 min, followed by 50 cycles of $95^{\circ} \mathrm{C}$ for $10 \mathrm{~s}$, $60{ }^{\circ} \mathrm{C}$ for $10 \mathrm{~s}, 72{ }^{\circ} \mathrm{C}$ for $15 \mathrm{~s}$. A negative control for each primer pair was also performed and at least three replicates of RNA isolation and cDNA synthesis were conducted. Melting curves and $\mathrm{Ct}$ value were analyzed to quantify gene abundance, $2^{-\Delta \Delta} C_{\mathrm{t}}$ method $(0 \mathrm{~d}$ was set as 1).

Transient overexpression and lignin measurement. To verify Ej4CL1 function in lignin biosynthesis, transient overexpression was conducted with $N$. tabacum leaves. The full length of Ej4CL1 was inserted into pGreen II 0029 62-SK vector [empty vector (EV)] (Hellens et al., 2005). The constructs were electroporated into Agrobacterium tumefaciens GV3101 (MP90) and stored in glycerol at $-80{ }^{\circ} \mathrm{C}$ for further use. Before formal injection, Agrobacterium was activated by streaking on a Luria-Bertani plate for $2 \mathrm{~d}$ and re-streaking on a new LuriaBertani plate for another $1 \mathrm{~d}$. Agrobacterium cultures were then suspended in infiltration buffer (10 mM MES, $10 \mathrm{~mm} \mathrm{MgCl}_{2}$, and $0.5 \mathrm{~mm}$ acetosyringone) and $\mathrm{OD}_{600}$ adjusted to 0.75 . Target genes and negative controls (EV SK) were injected on each side of the midrib of the same leaf. The infiltrated leaves were sampled $5 \mathrm{~d}$ after infiltration for further lignin analysis. Lignin content of flesh and $N$. tabacum were measured as described in our previous publications (Shan et al., 2008;

Table 1. Sequences of the primers used for gene isolation.

\begin{tabular}{|c|c|c|c|}
\hline Genes & Method used & Primers & Sequences $\left(5^{\prime}\right.$ to $\left.3^{\prime}\right)$ \\
\hline \multirow[t]{2}{*}{$\overline{E j C 4 H 1}$} & $3^{\prime} \mathrm{RACE}$ & Primary PCR & CTCCTCCGCATGGGGCAGTGCAA \\
\hline & & Secondary PCR & GCACATTCTAAGCCGACCTAGAGACC \\
\hline \multirow[t]{2}{*}{$\mathrm{EjC} 4 \mathrm{H} 2$} & $3^{\prime} \mathrm{RACE}$ & Primary PCR & CCACGTCGTTCACAACATCAACGCCG \\
\hline & & Secondary PCR & CCTCGGCCGCGGAGTACAAATCACA \\
\hline \multirow[t]{2}{*}{ Ej4CL8 } & 3'RACE & Primary PCR & ACACCCAGAAATTACCCTCCCCACGG \\
\hline & & Secondary PCR & TCATCTTCTCACACCAGCACACCGG \\
\hline \multirow[t]{4}{*}{ Ej4CL9 } & 3'RACE & Primary PCR & ACCTCATTGCTCAGAAGACCGGCGC \\
\hline & & Secondary PCR & GTGCCGAGTTCGTCTTCGCCTTC \\
\hline & 5'RACE & Primary PCR & CGGCAGTGTAAAAGGGGTTGGCGGT \\
\hline & & Secondary PCR & GAAGGCGAAGACGAACTCGGCAC \\
\hline \multirow[t]{4}{*}{ Ej4CL10 } & $3^{\prime} \mathrm{RACE}$ & Primary PCR & CCCCGCCAGCTCCAATCCCGAAATC \\
\hline & & Secondary PCR & ACTGGGAGGGTCAAAGGCGTGGAGT \\
\hline & 5'RACE & Primary PCR & GTGCTTCACCAAGGCCACGACCGC \\
\hline & & Secondary PCR & ATCGAACCTTCCCATGAACGCCAGC \\
\hline
\end{tabular}

Table 2. Sequences of the primers used for real-time polymerase chain reaction.

\begin{tabular}{lll}
\hline Genes & \multicolumn{1}{c}{ Forward primer $\left(5^{\prime}\right.$ to $\left.3^{\prime}\right)$} & \multicolumn{1}{c}{ Reverse primer $\left(5^{\prime}\right.$ to $\left.3^{\prime}\right)$} \\
\hline$E j P A L 1$ & TGAGATCACCAGGGGAGGAA & GAGCACCATTCCACTCCTTCA \\
$E j P A L 2$ & GGAGTTGGGAGGAGAGTACC & ACGATGATGCAGGACTCGAG \\
$E j C 4 H 1$ & AATTTAGGTCGAGGGAGGCG & CACCGAGGGATGTAAAGGCC \\
$E j C 4 H 2$ & ACCGTTGTTATGAAGCAGACG & AGCTGGAGAAGGATTGTGCT \\
$E j 4 C L 1$ & AGTCACCGTCGGGCAAAA & CATGCCCTTCCTTCAAAATG \\
$E j 4 C L 2$ & CCTCATCCAAATCCCAGAAA & AATGCCATCTCTGCCGAAC \\
$E j 4 C L 3$ & TCGACCAACAACAACAACGC & GTGACTGTTGCTTCTTTGTG \\
$E j 4 C L 4$ & CCTTATCCACTGGTTCATCAAAA & ATCGAATGCAACGCTGAGA \\
$E j 4 C L 5$ & TTTTCCCTCACTTTCTCACCA & AAATATCGGGGAGCTTGGAC \\
$E j 4 C L 6$ & TGCAGGGAAAAGTGTTGAGG & AAACATCGCACATACTTTGGAG \\
$E j 4 C L 7$ & ACAATCTCATCCAATCGTCAGT & TGTGATGTCGAGGGAGTAGT \\
$E j 4 C L 8$ & AAACAGAGCACTCCGGATCA & AGGAATCAATGAGGGCAGGT \\
$E j 4 C L 9$ & GAAAAGACCTCAGAGCCAAGC & GGCTCGCCCTCCAAATTG \\
$E j 4 C L 10$ & AAGTACCCATGGCCTTCGTC & TTGATGAATGCTACGCGTCG \\
$E j 4 C L 11$ & TGTGACACAGCTGCAATTGT & AACCAACCTACACCCACACA \\
\hline
\end{tabular}

Wang et al., 2010). Data were expressed on a fresh weight basis and all measurements were made in triplicate.

Statistical analysis. The statistical significance of differences was calculated using Student's $t$ test. Least significant differences $\left(\mathrm{LSD}_{0.05}\right)$ were calculated using DPS7.05 (Zhejiang University, Hangzhou, China).

\section{Results}

Enzyme activity changes in response to different treatments. In our previous report, HT and LTC treatments significantly alleviated 'LYQ' loquat lignification ( $\mathrm{Xu}$ et al., 2014). Using the same materials, PAL, C4H, and $4 \mathrm{CL}$ activities were measured. As shown in Fig. 1, activities of the three enzymes were all positively correlated with fruit lignification. Activities of PAL and 4CL were induced by low temperature $\left(0{ }^{\circ} \mathrm{C}\right)$ and peaked at $1 \mathrm{~d}$ and $4 \mathrm{~d}$, respectively, and these increases were significantly inhibited by LTC and HT. For example, PAL activity increased from 159 to $251 \mathrm{U} / \mathrm{mg}$ protein between days 0 and 1 , and decreased to 112 and $168 \mathrm{U} / \mathrm{mg}$ protein in LTC and HT treated

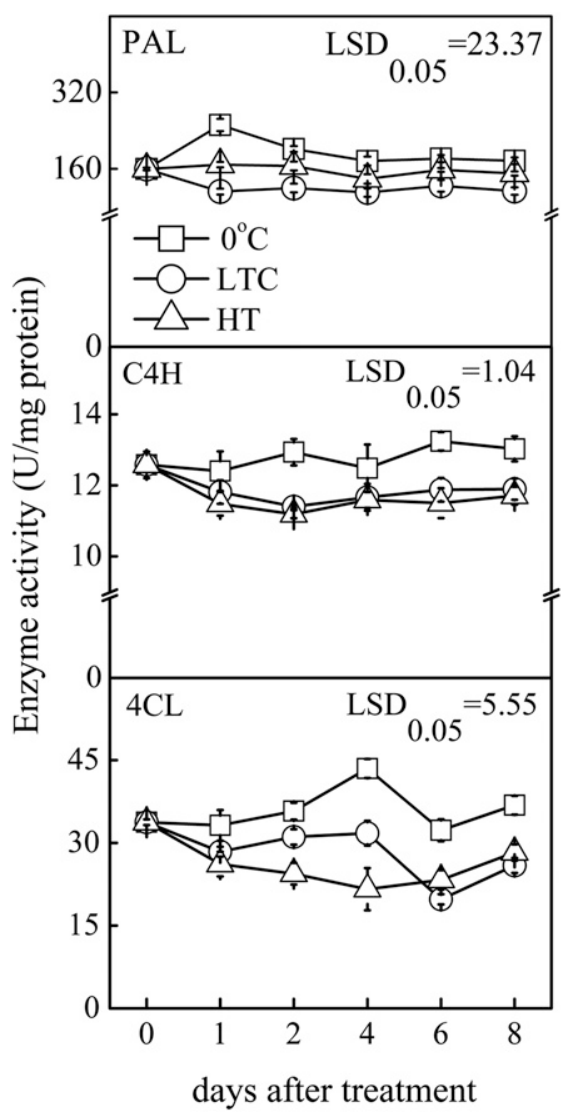

Fig. 1. Effects of HT and LTC treatments on Lphenylalanine ammonia-lyase (PAL), cinnamate 4-hydroxylase $(\mathrm{C} 4 \mathrm{H})$, and 4-coumarate: coenzyme ligase (4CL) activities in LYQ fruit. $\mathrm{HT}$ fruit was treated at $40{ }^{\circ} \mathrm{C}$ (in air) for $4 \mathrm{~h}$ and then was transferred to $0{ }^{\circ} \mathrm{C}$; LTC fruit were stored at $5^{\circ} \mathrm{C}$ for $6 \mathrm{~d}$ and then was transferred to $0{ }^{\circ} \mathrm{C}$. Error bars indicate se for three replicates. Least significant differences (LSDS) represent least significant difference at 0.05 . 
fruit, respectively, at $1 \mathrm{~d}$ (Fig. 1). Unlike PAL and $4 \mathrm{CL}, \mathrm{C} 4 \mathrm{H}$ activities remained constant during low-temperature storage, but also decreased in response to LTC and HT, thus also

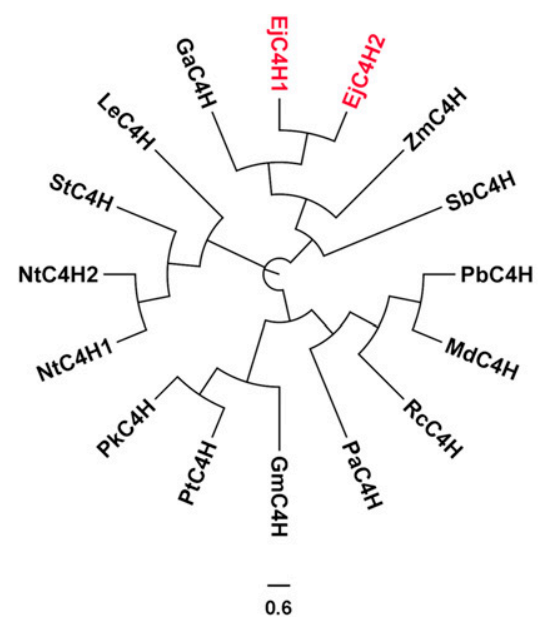

Fig. 2. Phylogenetic tree of cinnamate 4-hydroxylase $(\mathrm{C} 4 \mathrm{H})$ from loquat and other plants. Loquat EjC4Hs are highlighted in red. The NCBI accession numbers of the protein sequences are as follows: Genlisea aurea GaC4H (EPS65721); Lithospermun erythrorhizon LeC4H (BAB71717); Malus domestica MdC4H (AAY87450); Prunus avium PaC4H (AEA02458); Pyrus bretschneideri $\mathrm{PbC} 4 \mathrm{H}$ (AEK94316); Rubus coreanus RcC4H (ABX74779); Sorghum bicolor $\mathrm{SbC4H}$ (XP002461939); Solanum tuberosum StC4H (DQ341174); Zostera marina ZmC4H (KMZ73596); Glycine max GmC4H (FJ770486); Nicotiana tabacum NtC4H1 (DQ350352), $\mathrm{NtC} 4 \mathrm{H} 2$ (DQ350353).

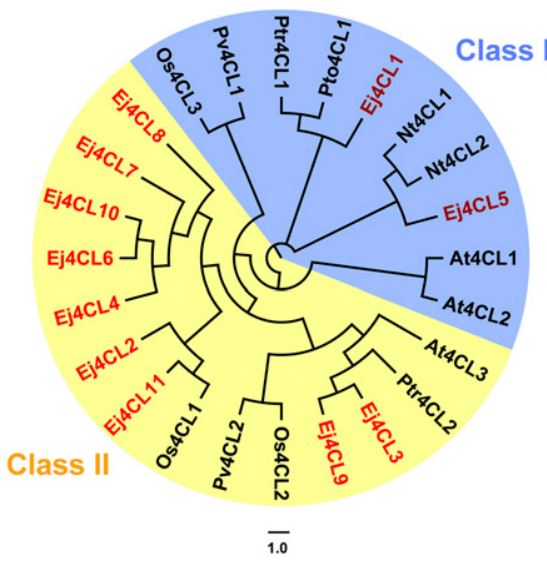

Fig. 3. Phylogenetic tree of 4-coumarate:coenzyme ligase (4CL) from loquat and other plants. Loquat Ej4CLs are highlighted in red. The NCBI accession numbers of the protein sequences are as follows: Glycine max Gm4CL1 (AAL98709), Gm4CL2 (P31687), Gm4CL3 (AAC97389); Nicotiana tabacum Nt4CL1 (O24145), Nt4CL2 (O24146); Arabidopsis thaliana At4CL1 (NP_175579), At4CL2 (NP_188761), At4CL3 (NP_176686); Populus tremuloides Ptr4CL1-2 (AAC24503-4); Populus tomentosa Pto4CL1 (AAL02145.1); Lolium perenne Lp4CL1-3 (AAF37732-4); Oryza sativa Os4CL1(BAD05189), Os4CL2 (Q42982), Os4CL3 (AB234050). to some extent correlated with fruit lignification (Fig. 1).

Gene isolation and analysis. As PAL, $\mathrm{C} 4 \mathrm{H}$, and 4CL all appeared to be associated with loquat fruit lignification at the biochemical level, their encoding genes were isolated, including EjPAL1-2 (EF685343-4) and Ej4CL1-5 (EF685345, KF767455-8) (Shan et al., 2008; Xu et al., 2014), on the basis of previous reports, and two novel $E j C 4 H$ (EjC4H1, KU176088; EjC4H2, KU176089) and six new Ej4CL genes (Ej4CL6-11, KU176090-KU176095) were isolated on the basis of RNA-seq and RACE.

Phylogenetic analysis revealed that $\mathrm{EjC} 4 \mathrm{HI}$ and $\mathrm{EjC} 4 \mathrm{H} 2$ were clustered with Genlisea aurea $\mathrm{GaC4H}$ (EPS65721) and Zostera marina ZmC4H (KMZ73596) (Fig. 2). Eleven Ej4CL genes, including five previously reported and six newly isolated, were divided into two major classes, class I and class II. Ej4CLI and Ej4CL5 belong to class I and were clustered with Pro4CL1, Pto4CL1, Nt4CL1, and Nt4CL2; whereas the other $E j 4 C L$ genes belong to class II (Fig. 3).

Expression of EjPAL, EjC4H, and Ej4CL genes in response to LTC and HT treatments. The relationship between previously isolated $E j P A L$ and Ej4CL genes and CI-related loquat lignification had not been investigated (Shan et al., 2008), thus the expression of all $E j P A L, E j C 4 H$, and Ej4CL genes was analyzed in response to low temperature, LTC and HT. Expression of EjPAL1 and EjPAL2 increased at $0{ }^{\circ} \mathrm{C}$ and this was significantly inhibited by HT treatment. However, LTC treatment had little effect on either EjPALI or EjPAL2 (Fig. 4).

Two $E j C 4 H$ genes, $E j C 4 H 1$ and $E j C 4 H 2$, exhibited different expression patterns, with the latter showing a much greater increase during LTC than the control. Expression of EjC4H1 slightly decreased during 1-3 d at $0{ }^{\circ} \mathrm{C}$ and increased to initial level of $0 \mathrm{~d}$ at 6 and $8 \mathrm{~d}$, whereas LTC and HT treatment had little effect on expression (Fig. 5). Expression of $\mathrm{EjC} 4 \mathrm{H} 2$ was increased 3 -fold in fruit stored for $2 \mathrm{~d}$ at $0{ }^{\circ} \mathrm{C}$ and 6 -fold in fruit stored $4 \mathrm{~d}$ after LTC treatment (Fig. 5).

Transcript accumulation patterns for the eleven Ej4CL genes could be generally divided into three groups: 1) expression of Ej4CL1, Ej4CL6, Ej4CL7, Ej4CL8 increased in response to $0{ }^{\circ} \mathrm{C}$; however, only Ej4CL1 and Ej4CL6 were significantly repressed by both LTC and HT; 2) Ej4CL9 had a much smaller response to $0{ }^{\circ} \mathrm{C}$, but was significant inhibited by LTC and HT; 3) other Ej4CL

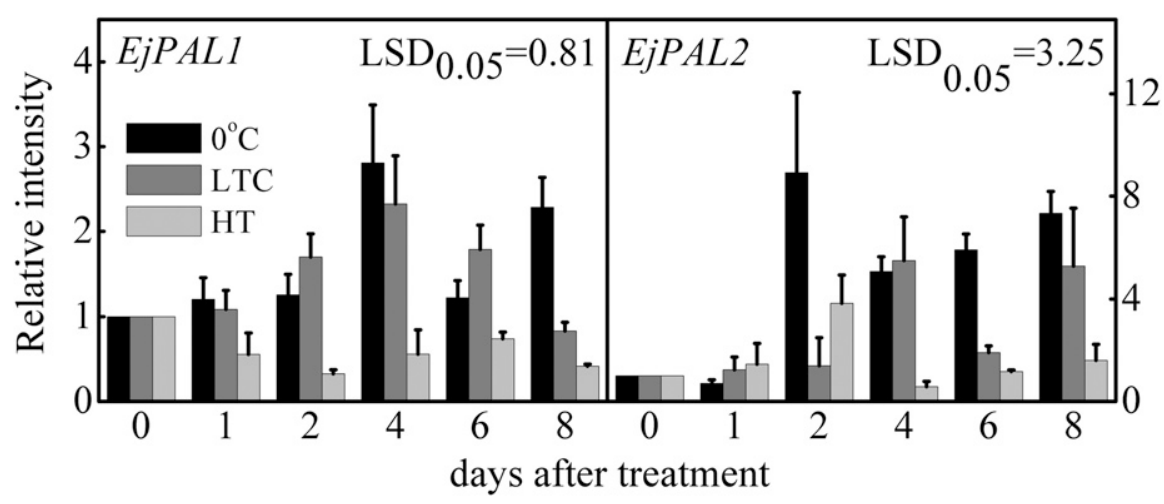

Fig. 4. Effects of low-temperature conditioning (LTC) and heat treatment (HT) on EjPAL expression. Transcripts of EjPAL genes were measured by real-time polymerase chain reaction. Day 0 fruit values were set as 1. Error bars indicate SE from three biological replicates. Least significant differences (LSDS) represent least significant difference at 0.05 .

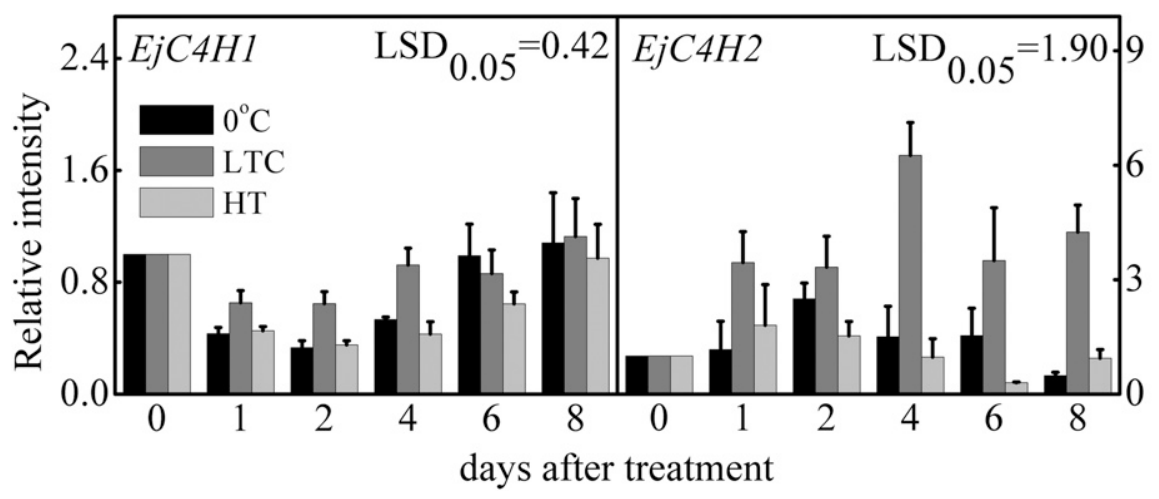

Fig. 5. Effects of low-temperature conditioning (LTC) and heat treatment (HT) on EjC4H expression. Transcripts of EjC4H genes were measured by real-time polymerase chain reaction. Day 0 fruit values were set as 1. Error bars indicate SE from three biological replicates. Least significant differences (LSDS) represent least significant difference at 0.05 . 

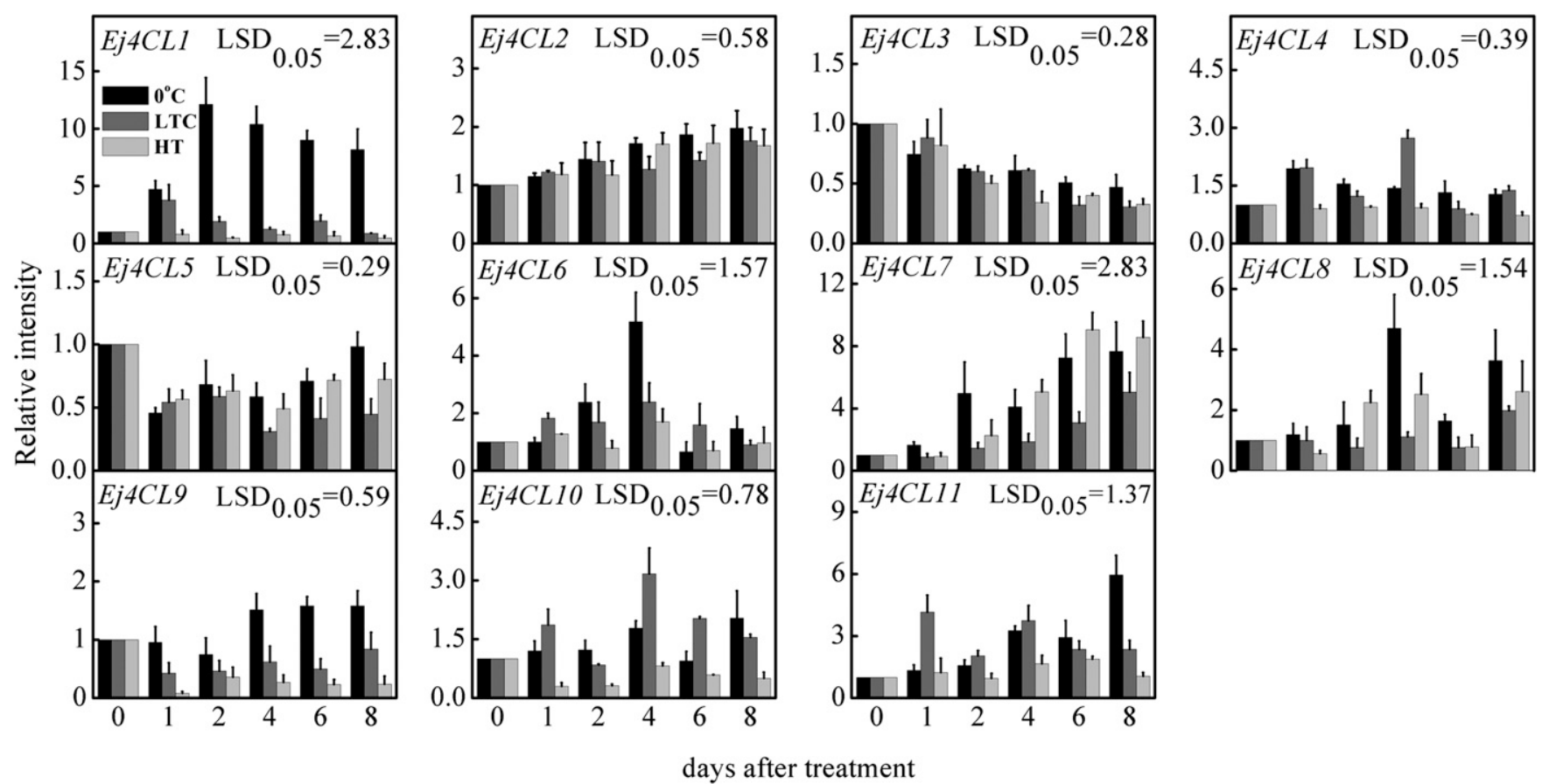

Fig. 6. Effects of low-temperature conditioning (LTC) and heat treatment (HT) on Ej4CL expression. Transcripts of Ej4CL genes were measured by real-time polymerase chain reaction. Day 0 fruit values were set as 1 . Error bars indicate sE from three biological replicates. Least significant differences (LSDs) represent least significant difference at 0.05 .

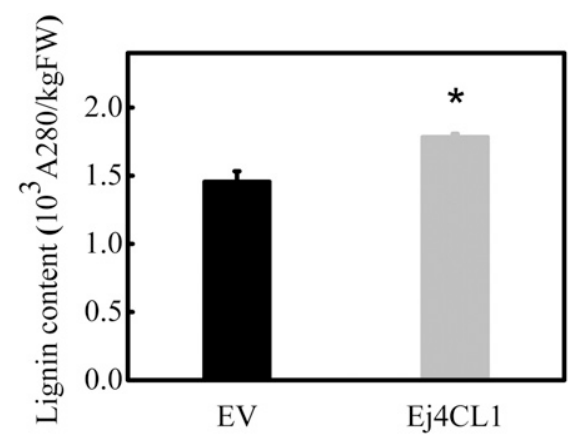

Fig. 7. Transient overexpression of Ej4CL1 in $N$. tabacum leaves. Ej4CL1 was driven by the CaMV 35S promoter. EV represents empty vector. Error bars indicate SE from three biological replicates $(* P<0.05)$.

genes showed little response and less difference between treatments. Of all the $E j 4 C L$ genes, Ej4CL1 was the most responsive, showing an $\approx 12$-fold increase after $2 \mathrm{~d}$ storage at $0{ }^{\circ} \mathrm{C}$ (Fig. 6).

Transient overexpression of Ej4CL1 in N. tabacum. The results of enzyme activity changes, phylogenetic tree and gene expression analysis all supported the putative role of Ej4CL1 in loquat fruit lignification. As loquat is a perennial fruit, making transgenic experiments difficult, the function of Ej4CL1 was tested in $N$. tabacum. Here, full length Ej4CL1 was cloned into pGreen II 0029 62SK vector, transferred to Agrobacterium tumefaciens GV3101, and injected into leaves of $N$. tabacum. The results indicated that leaves (half-blade) infiltrated with Ej4CL1 had a lignin content of $1.78 \times 10^{3}$
A280 $\mathrm{kg}^{-1}$ of fresh weight, which was significant higher than that of leaves treated with the EV, with lignin content of $1.45 \times 10^{3}$ A2 $80 \mathrm{~kg}^{-1}$ of fresh weight (Fig. 7).

\section{Discussion}

Lignin biosynthesis has been extensively studied in the model plant Arabidopsis and woody plants (Baucher et al., 1996; Voo et al., 1995), because of its roles in providing mechanical support, as a contaminant in paper manufacture, and as a substance adversely impacting on bioconversion and energy production from plants (Carroll and Somerville, 2009; Xu et al., 2011). In the past decade, the influence of lignin accumulation on fruit quality has been studied in various fruit species, such as loquat (Cai et al., 2006a, 2006b), where flesh lignification can be severe at low-temperature storage. A few biosynthetic enzymes in the phenylpropanoid pathway have been reported to be involved in loquat lignification, for instance PAL and CAD were associated with senescence-related lignification in loquat fruit (Cai et al., 2006a) and the activity of CAD was also related to the lignin content in different cultivars of loquat fruit (Shan et al., 2008). However, the role of other enzymes, such as $\mathrm{C} 4 \mathrm{H}$, have been generally ignored in previous research on CI and only EjCADl (Shan et al., 2008) and EjCCoAOMT (Liu et al., 2015) have been reported to be related to lignification associated with loquat fruit CI.

Here, three enzymes of the phenylpropanoid pathway were investigated. Enzyme activities of PAL, C4H, and 4CL were all positively correlated with loquat fruit
CI-induced lignification, and their activities were significantly repressed by two treatments (LTC and HT), which cause reduced lignification (Xu et al., 2014). This suggested that additional enzymes within the phenylpropanoid pathway were involved in loquat fruit lignification, although some enzymes, such as PAL and 4CL, have previously been considered as having no correlation with lignin contents of different cultivars (Shan et al., 2008). Accordingly, the expression patterns of 16 genes coding for PAL, C4H, and 4CL were analyzed. Accumulation of mRNAs for Ej4CL1, Ej4CL6 and Ej4CL9 were positively correlated with loquat fruit lignification in response to both LTC and HT; EjPAL1 only responded to HT treatment, but not LTC; while the remaining genes showed little association with fruit lignification. The present results indicate that expression of EjPAL1, EjPAL2, EjC2H1, and EjC2H4 showed limited correlation with activities of PAL and $\mathrm{C} 2 \mathrm{H}$, which suggest the potential of the other coding genes exist but remain unknown for loquat fruit, due to the lack of genome information. It is worth emphasizing that Ej4CL1 was most responsive to low temperature ( $>10$ folds) and also showed the most significant responses to HT and LTC treatments. Ej4CL1 was clustered with class I $4 C L$ genes, although class I and class II $4 C L$ genes have previously been considered as lignin specific, such as Ptr4CL1 (Hu et al., 1998). Taken together, the enzyme activity, phylogenetic tree and gene expression data, indicate that Ej4CL1 is a key candidate gene for understanding the regulation of CI-induced lignification in loquat fruit. 
Transient overexpression systems are widely used in various perennial fruit for gene function analysis, such as apple and crabapple anthocyanin-related genes (Espley et al., 2007); persimmon tannin-related genes (Min et al., 2012; Mo et al., 2015), kiwifruit aroma-related genes (Nieuwenhuizen et al., 2015). Using similar technologies, function of Ej4CL1 on lignin biosynthesis was verified in N. tabacum. Transient overexpression of Ej4CL1 significantly triggered lignin accumulation, which further supported that loquat Ej4CL1 is an important candidate for lignin biosynthesis.

In conclusion, $\mathrm{PAL}, \mathrm{C} 4 \mathrm{H}$ and $4 \mathrm{CL}$ were positively associated with loquat fruit CIinduced lignification in addition to CCOAOMT and $\mathrm{CAD}$, reported by others. Moreover, Ej4CL1, a class I member, was characterized as a candidate gene for control of lignin biosynthesis, whereas several other members of this gene family are not involved in this response.

\section{Literature Cited}

Barros, J., H. Serk, I. Granlund, and E. Pesquet. 2015. The cell biology of lignification in higher plants. Ann. Bot. (Lond.) 115:1053-1074.

Baucher, M., B. Chabbert, G. Pilate, J. Doorsselaere, M.T. Tollier, M. Petit-Conil, D. Cornu, B. Monties, M. Montagu, D. Inzé, L. Jouanin, and W. Boerjan. 1996. Red xylem and higher lignin extractability by down-regulating a cinnamyl alcohol dehydrogenase in poplar (Populus tremula x P. alba). Plant Physiol. 112: 1479-1490.

Bonawitz, B.D. and C. Chapple. 2012. The genetics of lignin biosynthesis: Connecting genotype to phenotype. Annu. Rev. Genet. 44:337-363.

Bradford, M.M. 1976. A rapid and sensitive method for the quantitation of microgram quantities of protein utilizing the principle of protein-dye binding. Anal. Biochem. 282:152160.

Cai, C., C.J. Xu, X. Li, I.B. Ferguson, and K.S. Chen. 2006a. Accumulation of lignin in relation to change in activities of lignification enzymes in loquat fruit flesh after harvest. Postharvest Biol. Technol. 40:163-169.

Cai, C., C.J. Xu, L.L. Shan, X. Li, C.H. Zhou, W.S. Zhang, I.B. Ferguson, and K.S. Chen. 2006b. Low temperature conditioning reduces postharvest chilling injury in loquat fruit. Postharvest Biol. Technol. 41:252-259.

Cai, C., W.P. Xu, W.S. Zhang, X. Li, I.B. Ferguson, and K.S. Chen. 2006c. Effect of 1-MCP on postharvest quality of loquat fruit. Postharvest Biol. Technol. 40:155-162.

Cai, Y.T., S.F. Cao, Z.F. Yang, and Y.H. Zhang. 2011. MeJA regulates enzymes involved in ascorbic acid and glutathione metabolism and improves chilling tolerance in loquat fruit. Postharvest Biol. Technol. 59:324-326.

Cao, S.F., Y.T. Cai, Z.F. Yang, and Y.H. Zheng. 2012. MeJA induces chilling tolerance in loquat fruit by regulating proline and $\mathrm{Y}$-aminobutyric acid contents. Food Chem. 133:1466-1470.

Carroll, A. and C. Somerville. 2009. Cellulosic biofuels. Annu. Rev. Plant Biol. 60:165-182.

Espley, R.V., R.P. Hellens, J. Putterill, D.E. Stevenson, S. Kutty-Amma, and A.C. Allan. 2007. Red colouration in apple fruit is due to the activity of the MYB transcription factor, MdMYB10. Plant J. 49:414-427.

Hellens, R.P., A.C. Allan, E.N. Friel, K. Bolitho, K. Grafton, M.D. Templeton, S. Karunairetnam, A.P. Gleave, and W.A. Laing. 2005. Transient expression vectors for functional genomics, quantification of promoter activity and RNA silencing in plants. Plant Methods 1:13.

Hu, W.J., A. Kawaoka, C.J. Tsai, J. Lung, K. Osakabe, H. Ebinuma, and V.L. Chiang. 1998. Compartmentalized expression of two structurally and functionally distinct 4coumarate:CoA ligase genes in aspen (Populus tremuloides). Proc. Natl. Acad. Sci. USA 95:5407-5412.

Knobloch, K.H. and K. Hahlbrock. 1975. Isoenzymes of p-coumarate: CoA ligase from cell suspension cultures of Glycine max. Eur. J. Biochem. 52:311-320.

Koukol, J. and E.E. Conn. 1961. The metabolism of aromatic compounds in higher plants. IV. Purification and properties of the phenylalanine deaminase of Hordeum vulgare. J. Biol. Chem. 236:2692-2698.

Lamb, C.J. and P.H. Rubery. 1975. A spectrophotometric assay for trans-cinnamic acid 4hydroxylase activity. Anal. Biochem. 68: 554-561.

Lee, D., K. Meyer, C. Chapple, and C.J. Douglas. 1997. Antisense suppression of 4-Coumarate: Coenzyme A ligase activity in Arabidopsis leads to altered lignin subunit composition. Plant Cell 9:1985-1998.

Li, X., C.J. Xu, S.S. Korban, and K.S. Chen. 2010. Regulatory mechanisms of textural changes in ripening fruits. Crit. Rev. Plant Sci. 29:222243.

Lin, S., R.H. Sharpe, and J. Janick. 1999. Loquat: Botany and horticulture. Hort. Rev. 23:233276.

Liu, Y.X., D.M. Zou, B.S. Wu, D.H. Lin, Z.H. Zhang, and J.C. Wu. 2015. Cloning and expression analysis of a CCOAOMT homolog in loquat fruit in response to low-temperature storage. Postharvest Biol. Technol. 105: $45-50$.

Min, T., X.R. Yin, Y.N. Shi, Z.R. Luo, Y.C. Yao, D. Grierson, I.B. Ferguson, and K.S. Chen. 2012. Ethylene responsive transcription factors interact with promoters of $A D H$ and $P D C$ involved in persimmon (Diospyros kaki) fruit deastringency. J. Expt. Bot. 63:6393-6405.

Mo, R.L., Y.M. Huang, S.C. Yang, Q.L. Zhang, and Z.R. Luo. 2015. Development of Agrobacterium-mediated transient transformation in persimmon (Diospyros kaki Thunb.). Sci. Hort. 192:29-37.

Nieuwenhuizen, N.J., X.Y. Chen, M.Y. Wang, A.J. Matich, R.L. Perez, A.C. Allan, S.A. Green, and R.G. Atkinson. 2015. Natural variation in monoterpene synthesis in kiwifruit: Transcriptional regulation of terpene synthases by NAC and
ETHYLENE-INSENSITIVE3-Like transcription factors. Plant Physiol. 167:1243-1258.

Sewalt, V.J.H., W. Ni, J.W. Blount, H.G. Jung, S.A Masoud, P.A. Howles, C. Lamb, A. Sameer, A. Paul, C. Lamb, and R.A. Dixon. 1997. Reduced lignin content and altered lignin composition in transgenic tobacco down-regulated in expression of L-phenylalanine ammonia-lyase or cinnamate 4-hydroxylase. Plant Physiol. 115:41-50.

Shan, L.L., X. Li, P. Wang, C. Cai, B. Zhang, C.D. Sun, W.S. Zhang, C.J. Xu, I.B. Ferguson, and K.S. Chen. 2008. Characterization of cDNAs associated with lignification and their expression profiles in loquat fruit with different lignin accumulation. Planta 227:1243-1254.

Vanholme, R., B. Demedts, K. Morreel, J. Ralph, and W. Boerjan. 2010. Lignin biosynthesis and structure. Plant Physiol. 153:895-905.

Voo, K.S., R.W. Whetten, D.M. O'Malley, and R.R. Sederoff. 1995. 4-Coumarate:coenzyme A ligase from loblolly pine xylem (isolation, characterization, and complementary DNA cloning). Plant Physiol. 108:85-97.

Wang, P., B. Zhang, X. Li, C.J. Xu, X.R. Yin, L.L. Shan, I.B. Ferguson, and K.S. Chen. 2010. Ethylene signal transduction elements involved in chilling injury in non-climacteric loquat fruit. J. Expt. Bot. 61:179-190.

Xu, B., L.L. Escamilla-Treviño, N. Sathitsuksanoh, Z.X. Shen, H. Shen, Y.H.P. Zhang, R.A. Dixon, and B.Y. Zhao. 2011. Silencing of 4-coumarate:coenzyme A lignase in switchgrass leads to reduced lignin content and improved fermentable sugar yields for biofuel production. New Phytol. 192:611-625.

Xu, M.J., J.F. Dong, M. Zhang, X.B. Xu, and L.N. Sunday. 2012. Cold-induced endogenous nitric oxide generation plays a role in chilling tolerance of loquat fruit during postharvest storage. Postharvest Biol. Technol. 65:5-12.

Xu, Q., X.R. Yin, J.K. Zeng, H. Ge, M. Song, C.J. Xu, X. Li, I.B. Ferguson, and K.S. Chen. 2014. Activator- and repressor-type MYB transcription factors are involved in chilling injury induced flesh lignification in loquat via their interactions with the phenylpropanoid pathway. J. Expt. Bot. 65:4349-4359.

Xu, Q., W.W. Wang, J.K. Zeng, J. Zhang, D. Grierson, X. Li, X.R. Yin, and K.S. Chen. 2015. A $N A C$ transcription factor, EjNACl, affects lignification of loquat fruit by regulating lignin. Postharvest Biol. Technol. 102:25-31.

Zeng, J.K., X. Li, Q. Xu, J.Y. Chen, X.R. Yin, I.B. Ferguson, and K.S. Chen. 2015. EjAP2-1, an $A P 2 / E R F$ gene, is a novel regulator of fruit lignification induced by chilling injury, via interaction with $E j M Y B$ transcription factors. Plant Biotechnol. J. 13:1325-1334.

Zeng, J.K., X. Li, J. Zhang, H. Ge, X.R. Yin, and K.S. Chen. 2016. Regulation of loquat fruit low temperature response and lignification involves interaction of heat shock factors and genes associated with lignin biosynthesis. Plant Cell Environ. 39:1780-1789.

Zhao, Q. and R.A. Dixon. 2011. Transcriptional networks for lignin biosynthesis: More complex than we thought? Trends Plant Sci. 16:227-233. 